\title{
Identification of Genotype Specific Marker for Samba Mahsuri (BPT 5204) Variety
}

\author{
K. Aruna Kumari ${ }^{*}$, A. Prasad babu ${ }^{2}$, Ch.V. Durga Rani ${ }^{1}$ and R.M. Sundaram ${ }^{2}$ \\ ${ }^{1}$ Department of Agricultural Biotechnology, College of Agriculture, Rajendranagar, Acharya, \\ N G Ranga Agricultural University, Rajendranagar, Hyderabad 500 030, India \\ ${ }^{2}$ Crop Improvement Section, Directorate of Rice Research, Rajendrangar, \\ Hyderabad 500 030, India \\ *Corresponding author
}

\section{A B S T R A C T}

\section{Keywords}

Samba Mahsuri, SSRs and Multiplex PCR.

Article Info

Accepted:

17 November 2018

Available Online:

10 December 2018
Samba Mahsuri is an elite, fine grain rice variety, which is grown extensively in India. To finger print and identify a genotype specific marker from 26 other closely related fine grain types, we utilized a 113 SSR markers distributed throughout the rice genome. Twenty six markers found to be polymorphic among the rice varieties analyzed. A twin marker combination RM 19426 +JGT 11-16.3 could unambiguously differentiate samba Mahsuri from other fine grain varieties through multiplex PCR.

\section{Introduction}

About $25 \%$ area under cultivation of rice in the country is being occupied by different varieties developed by Acharya N G Ranga Agricultural University (ANGRAU), Hyderabad, India and contributing to $1 / 3^{\text {rd }}$ of country's rice production. Three out of the five best varieties in the country viz., Samba Mahsuri (BPT 5204), Swarna (MTU 7029) and Vijetha (MTU 1001) were released by ANGRAU. The variety, Samba Mahsuri is extremely popular amongst rice farmers and consumers because of its high yield, medium- slender, fine-grain type and excellent cooking and eating quality. This variety is being targeted for improvement of multiple traits like biotic and abiotic stress tolerance/resistance. The original variety along with its improved versions needs to be protected in terms of plant breeder's right in the present era of intellectual property protection. There is an imminent need to identify genotype specific markers for cultivar identity and trueness of elite varieties like Samba Mahsuri so that such molecular markers can be utilized to unambiguously identify the variety and purity of seed-lots of 
the variety. During the seed production chain, the variety can get contaminated with other medium-slender varieties (some of which are cultivated extensively in India) and such impurities may go on accumulating unnoticed, finally leading to deterioration of the genetic quality of the variety. SSRs have been extensively used in rice for molecular fingerprinting and assessment of genetic purity of hybrids and parental lines 10,18,20. Based on the above, we designed the present study with the following objective to identify genotype specific marker(s) for Samba Mahsuri in order to differentiate it from other popular fine-grain varieties.

\section{Materials and Methods}

\section{Rice genotypes}

A total of twenty seven genotypes including Samba Mahsuri (Table 1), which were developed and released by ANGRAU were analyzed for the identification of genotypic specific molecular finger prints SSR marker.

\section{DNA extraction and polymerase chain reaction}

Total genomic DNA was extracted from freshly germinated young seedlings. A total of 87 hyper-variable SSR primer pairs (selected from http://www.gramene.org) and 26 $(\text { GATA })_{n}$ motif specific SSR primer pairs (Rajendra Kumar et al., 2009) distributed across the 12 rice chromosomes were used for PCR amplification (listed in supplementary Table 1). DNA samples (50 ng) were amplified in $10 \mu 1$ reaction volumes containing 1X PCR buffer [10 mM Tris- $\mathrm{HCl}(\mathrm{pH} 8.3), 50$ $\mathrm{mM} \mathrm{KCl}, 1.5 \mathrm{mM} \mathrm{MgCl} 2,0.01 \%$ (v/v) gelatin] (Bangalore Genei, India), $0.2 \mathrm{mM}$ of each dNTPs (Bangalore Genei, India), 10 pmol of each primer and $1 \mathrm{U}$ of Taq polymerase (Bangalore Genei, India). PCR was carried out in a Thermal cycler (PerkinElmer, USA). A PCR profile consisting of was
5 min initial denaturation at $94^{\circ} \mathrm{C}, 35$ cycles of $1 \mathrm{~min}$ denaturation at $94^{\circ} \mathrm{C}, 1 \mathrm{~min}$ annealing at $55^{\circ} \mathrm{C}, 2 \mathrm{~min}$ extension at $72^{\circ} \mathrm{C}$ and $7 \mathrm{~min}$ at $72^{\circ} \mathrm{C}$ final extension was followed. The amplified products were resolved on $4 \%$ Metaphor agarose gels (Lonza, USA), stained with ethidium bromide and visualized under UV in a gel documentation system (Alpha Innotech, USA). The sizes of the amplified fragments were estimated with the help of Alphaease software utility of the gel documentation system using 50 and 100 bp DNA ladders (MBI Fermentas, Lithuania) as the molecular size standards. Initially an attempt was made to identify a single SSR marker-which could unambiguously differentiate Samba Mahsuri from the rest of the genotypes. Since no single marker could differentiate Samba Mahsuri from other varieties, combinations of two SSR markers which could be pre-PCR multiplexed were selected based on the amplification data of the SSR markers in order to identify genotype specific marker combinations. Only those markers that did not show significant primer sequence matches and those with amplicon sizes well separated from each other were selected for pre-PCR multiplexing.

\section{Results and Discussion}

DNA fingerprinting methods based on polymerase chain reaction have become methods of choice for germplasm characterization, diversity analysis and seed purity assays (Sundaram et al., 2008). A variety of DNA markers are now available for fingerprinting cultivars and for marker assisted selection. Even though the utility of RAPD fingerprints for establishing genotype identity is known in case of rice hybrids (Qian et al., 1996 and Wang et al., 1994), SSR markers are considered more reliable because of their ability to produce high-fidelity profiles as a result of their co-dominant nature and chromosome specificity (Nandakumar et al., 2004). 
Figure.1 Amplification pattern generated through multiplex PCR of JGT 11-16.3 and RM 19426

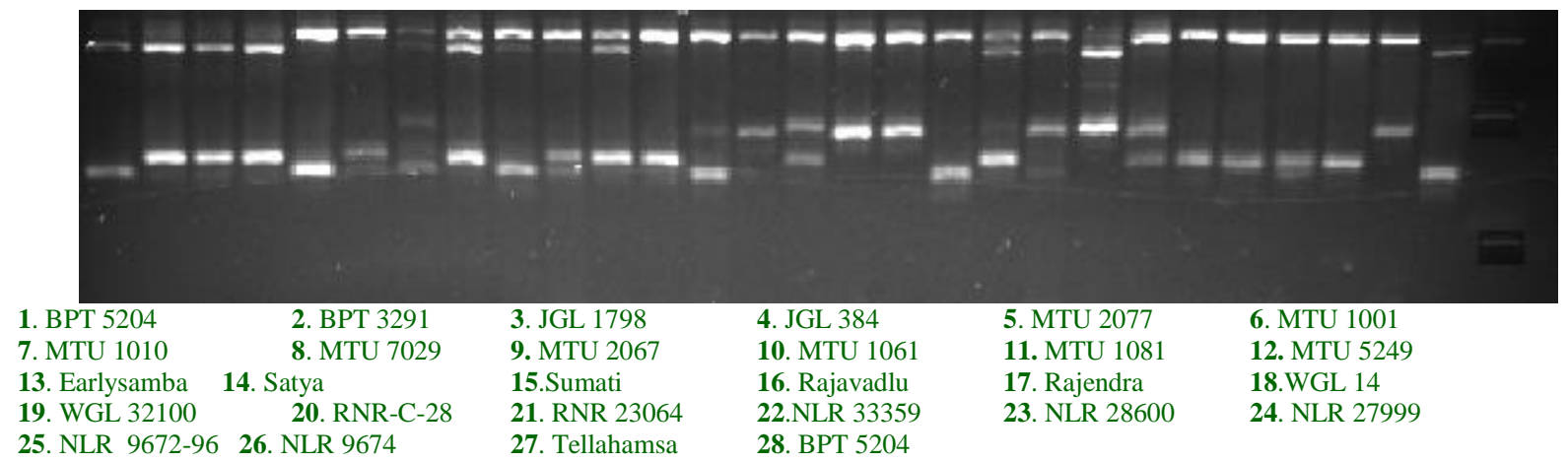

Table.1 Details of twenty seven rice varieties analyzed in the present study

\begin{tabular}{|c|c|c|c|c|}
\hline S No & Variety & Year of Release & Parentage & $\begin{array}{l}\text { Grain } \\
\text { type }\end{array}$ \\
\hline 1 & Samba Mahsuri (BPT 5204) & 1986 & GEB 24 / T(N)1 // Mahsuri & MS \\
\hline 2 & Sona Mahsuri (BPT 3291) & 1982 & Sona / Mahsuri & MS \\
\hline 3 & Jagtial Sannalu (JGL 1798) & 2002 & BPT 5204 / Kavya & MS \\
\hline 4 & Polasa Prabha (JGL 384) & 2002 & Kavya / BPT 5204 & MS \\
\hline 5 & Krishnaveni (MTU 2077) & 1989 & Sowbhagya /ARC 5984 & MS \\
\hline 6 & Vijetha (MTU 1001) & 1995 & MTU 5249 / MTU 7014 & MS \\
\hline 7 & Cottondora Sannalu (MTU 1010) & 2000 & Krishnaveni / IR 64 & LS \\
\hline 8 & Swarna (MTU 7029) & 1982 & Vasishtha / Mahsuri & MS \\
\hline 9 & Chaitanya (MTU 2067) & 1988 & Sowbhagya /ARC 5984 & MS \\
\hline 10 & Indra (MTU 1061) & 2007 & PLA1100/MTU1010 & MS \\
\hline 11 & MTU 1081 & Completed minikit trails & Ajaya/BPT 5204 & MS \\
\hline 12 & Vajram (MTU 5249) & 1986 & MTU 4569 /ARC 6650 & LS \\
\hline 13 & Early Samba (RNR M-7) & 2000 & Mutant of BPT 5204 & MS \\
\hline 14 & Satya (RNR 1446) & 1987 & Tellahamsa / Rasi & LS \\
\hline 15 & Sumati(RNR 18833) & 2002 & Chandan / Pak Basmati & LS \\
\hline 16 & Rajavadlu(RNR 99377) & 1993 & Rajendra / IR30 & LS \\
\hline 17 & Rajendra (RNR 12329) & 1976 & IJ52 / TN1 & MS \\
\hline 18 & Warangal samba (WGL 14) & 2005 & BPT 5204 /ARC 5984 // BPT 3291 & MS \\
\hline 19 & Warangal Sannalu (WGL 32100) & 2007 & Divya / BPT5204 & MS \\
\hline 20 & RNR -C-28 & Completed minikit trails & IR 64 / IET 9994 & LS \\
\hline 21 & Taramati (RNR 23064) & & BPT 5204 / Tellahamsa & MS \\
\hline 22 & Shravani (NLR-33359) & 2000 & Selection from IR 50 & LS \\
\hline 23 & Simhapuri (NLR-28600) & 1991 & RP 5-32 / Bulk H9 & SB \\
\hline 24 & Tikkana (NLR-27999) & 1988 & RP 31-49-2 / BCP 2 & SB \\
\hline 25 & Pinakini (NLR-9672-96) & 1987 & Bulk H 9 / Millekkunning & MS \\
\hline 26 & Kottamolagolukulu(NLR-9674) & 1982 & Bulk HG 9 / Millekkunning & SB \\
\hline 27 & Tellahamsa (C10754) & 1971 & HR 12 / T(N)1 & MS \\
\hline
\end{tabular}

MS: medium slender, LS: Long slender, SB: Short Bold 
Supplementary table.1 List of SSR markers used for identification of genotype specific marker for BPT 5204

\begin{tabular}{|c|c|c|c|c|}
\hline S. No & Marker & Chromosome & Forward primer sequence & Reverse primer sequence \\
\hline 1 & RM 10936 & 1 & ACGGTTTGGAAGTGTTCGTAGG & TGGTACTGCATAATCTCAGCATCG \\
\hline 2 & RM 11229 & 1 & TGACAGAAACAAAGCGGAAGG & TCCAAACCGCTATTCTTGTAGC \\
\hline 3 & RM 3917 & 1 & CGGACGACTCCGACAACACG & CGAACGAACGAGGACGAACG \\
\hline 4 & RM 17600 & 1 & CAGCATCAAGGGTCGCTACACG & CGATGGCGTGGGATAATTACGG \\
\hline 5 & RM 11278 & 1 & ACTTCTTGTAGCACTGCACCTTCG & CCTCGGCAACTGCTTCAAGG \\
\hline 6 & RM 10649 & 1 & GACCAATATGGAGTGGTCTCTTGG & ACGCTCCGCCTGCTATTTAGG \\
\hline 7 & RM 8126 & 1 & TGGGCCTCTTTGTTTCATACTCC & TCCTCATCTCTCTCCGTGTCTCC \\
\hline 8 & RM 11865 & 1 & CAACTCATTCGGCTTCTCTTTCC & GCAATTGCATGAGTTGGAATGG \\
\hline 9 & RM 6942 & 2 & CGAACTCCCAATTACAGATCACG & TCATCCAAGACTTAGGTGGAGAAGC \\
\hline 10 & RM 13155 & 2 & TACCAACAGGGAGTTGTCTCTCG & AGCGACGGTGTAAAGAATAAGTCG \\
\hline 11 & RM 6933 & 2 & AATGCCTAGCACTCATCCTTGC & AGGCACCCTACGATGAAATAGTGG \\
\hline 12 & RM 12469 & 2 & ACTCCATCGAACCCTGTTAGAGC & GTCCATGTTTGCTTACGTGTTTGC \\
\hline 13 & RM 13154 & 2 & GGTACTTAGCGTGCAACTTTAACC & TAGGTAACTAGACGAAGCGATAGAGG \\
\hline 14 & RM 13812 & 2 & AACACTAACCGGGACTAAAGATCG & CTGAGATAACGACTACTGCTACATCG \\
\hline 15 & RM 6938 & 2 & CCGATTAGCGATTGATATGGAGTAGG & AGTGCACAGCCATGGAATTATGC \\
\hline 16 & RM 14140 & 2 & ССТСССТСТССАAАСАСАTTGC & TCATCAGCAGACAGAATGTTGACC \\
\hline 17 & RM 15679 & 3 & TAGATGTATGAGTCGGAATGGAGTCG & CAGACGCAGTGTGTGTATGAAGTTCC \\
\hline 18 & RM 15630 & 3 & AACTCGAAGGATCTCGCCCAACC & ACCCACCTCCTCACGCTGTACG \\
\hline 19 & RM 14250 & 3 & GATTACTGCCGATTCGATAGC & AAATGGGACATGTTCTCTCG \\
\hline 20 & RM 14931 & 3 & GCTTCACGCACATATTGCCTTCC & ATCСТCССАСТCССAAATTATCTCC \\
\hline 21 & RM 15331 & 3 & GGTTCGGTGCTTTCTCTTTCAGC & AGCGATGCCGTCCTTACACC \\
\hline 22 & RM 5928 & 3 & CTTACCTTCTGAAATGGAGGTAGC & CAAGGTGAAAGACGAAGAATGC \\
\hline 23 & RM 15831 & 3 & AACACAATTCACCGGTCCTTAGC & ACGTGGGTGATCGTGTCTGC \\
\hline 24 & RM 15727 & 3 & ATCTGTGCGGACCACCATGC & CGCTACGAGCAGCGTACTTAAAGC \\
\hline 25 & RM 15466 & 3 & CGGAGTGATCATCAGAGTCG & GTAGAATCGCATGTAGAGTCTTAGGG \\
\hline 26 & RM 16153 & 3 & TGGTTGTGGTATAGCACGGTAAGC & TGACCCAAGGAGATACTAGGTTGC \\
\hline 27 & RM 15288 & 3 & GAGAGGCTCCTACTGCGGTTGC & CCTTTCCATCTCCTTGTTTCTCTGC \\
\hline 28 & RM 6759 & 3 & CCCGAGTCTTCATAGAGATATTCC & ATCCCTAGCTAGCCTTCCTTCC \\
\hline 29 & RM 14582 & 3 & ATAACCAAGATCTCGGCCCATCC & CCCATGTCACCGACAACTATCTAGC \\
\hline 30 & RM 14320 & 3 & CACCTGTAAATTAGGACACTGG & CAGTGTACTTTGAACTGCCTAGC \\
\hline 31 & RM 14860 & 3 & GGAAGGTGATTTCATCCGGTAGC & TGGCATGTTTAATGCTGGTTCG \\
\hline 32 & RM 16606 & 4 & TGCACTTCTTTAGAGTAGGAGGAAGC & CATGCATGTGTCCAAAGATTCG \\
\hline 33 & RM 16592 & 4 & CTTAGCACGGACACTCATATTTGG & CACAATACGTTTGATGGCTTGC \\
\hline 34 & RM 16801 & 4 & CGTTCAAGGAGCTTGTGTTGATCC & GGACCGATTTAAGTGAACGTTGATGG \\
\hline 35 & RM 17127 & 4 & CTCAATGTTTCCCACAGTTACCG & TGTGTTATGTGTGCGTGATGAGC \\
\hline 36 & RM 16652 & 4 & TGACATTAGTTGTGGCAGATCC & CCTAGAATCTCATCTGTCTTCTGG \\
\hline 37 & RM 16649 & 4 & CTCCCTTCATGCGTAAGCTCTCC & GCAAACAGGATCCTCCACAAAGG \\
\hline 38 & RM 16343 & 4 & AGAACCAGCAGTTTCTTTGC & TTCTATCCCTACAGTCTTGACAGC \\
\hline 39 & RM 16913 & 4 & GTGTACGTGTTGGCTCTCTGTACG & GATGTTGCTTGTGCTGCAACC \\
\hline 40 & RM 18222 & 5 & TGATTCCTCTATATGCAGCCTTGG & TATCGTGGTTTCATCGTGTGTGC \\
\hline 41 & RM 5592 & 5 & CTCGCTCTTACAACTTTCAAGC & САСТTАССТССАСТTСТСААСC \\
\hline 42 & RM 18857 & 5 & ACCGGTCCTTAGCTTCTGAGC & ACCAACCGGGACTAAAGATCG \\
\hline 43 & RM 18888 & 5 & GGTGCAAATGCTTGTAGTCCTATGC & GCACCACCAACCTAACAGTGAGC \\
\hline 44 & RM 18065 & 5 & CGATGGTGAGTGGTGATTCATGC & ATCATCCGCGCATTAGCATTTCC \\
\hline
\end{tabular}


Int.J.Curr.Microbiol.App.Sci (2018) 7(12): 2495-2501

\begin{tabular}{|c|c|c|c|c|}
\hline 45 & RM 20060 & 6 & CACACATGAGTGGTTAGGTAAGATGC & CAGTGACAAGAGCGAAATGATCC \\
\hline 46 & RM 20458 & 6 & GTGTGGTGGTCTTGGGATGTTGG & TATAGCCCAGCAAGGGTGGTACG \\
\hline 47 & RM 20196 & 6 & GTGGACCCACTACACAACATGG & TGTTCTACACTACGCCATTAGGC \\
\hline 48 & RM 19697 & 6 & AACAACCTGAGAACACCTCTTGG & GGACAAACACATGGTGATCTGC \\
\hline 49 & RM 20096 & 6 & CGGTAAGCCATAAATAGATCCCAAGG & TTTGAACAGCGACACGGTTTCC \\
\hline 50 & RM 19682 & 6 & CCCACTGATTGACCAAGAGC & TTTGCTAAAGGAGCACTACGC \\
\hline 51 & RM 19426 & 6 & CGGTGTCTTCTTTAAACAGC & ATGGATAAGCGGTATGTTCC \\
\hline 52 & RM 400 & 6 & TTACACCAGGCTACCCAAACTCG & TTGCTGAGTTCCCTCGTCTATCC \\
\hline 53 & RM 19660 & 6 & TTTGTCCCTGCCGTACTTGC & AGCCACGTTGGGTGAAATTAGC \\
\hline 54 & RM 20818 & 7 & AGATGCAGATAGATGCATGTCACG & ACCGATCATCCACGATCCTACG \\
\hline 55 & RM 6697 & 7 & TATTCCCGGGAGATCCAACAGC & AAGATCCAGTCGATTTGGTTCAGG \\
\hline 56 & RM 21069 & 7 & ATCTAGTACCGGATGTGACACG & AGACAGAGGCATGACAGAAAGG \\
\hline 57 & RM 5720 & 7 & GACTCGTCACTGACACTGATACG & CTTGTTAGGAAGAGCATTCTGC \\
\hline 58 & RM 21258 & 7 & TATCATTCCGGTCCAAAGTGTCG & TCCGGTCCAAAGTCTCATTTGC \\
\hline 59 & RM 21564 & 7 & CGACGGAAACACAATTCATAGG & ACCAACCGGGACTAAAGATCG \\
\hline 60 & RM 21813 & 7 & NA & NA \\
\hline 61 & RM 23237 & 8 & TAAAGCATTTGGACGGTGGATGG & GAGGTGGGTGTGACCCTTGG \\
\hline 62 & RM 22622 & 8 & TAGGCCGTTCTGACGTAATACCC & CAGTGATGGTGATGCGATTTAGC \\
\hline 63 & RM 22585 & 8 & CACCGATTATTGTCGTATGG & AGTGAGGAAGGGAAGAATACG \\
\hline 64 & RM 5933 & 8 & AGCGATTCAGAACGAATCAACG & TGCCAAAGCTACACAAATCTGACC \\
\hline 65 & RM 6699 & 8 & TGGTATCACCATTCACCAACACC & TCCTTACGCTACCACGACTTCC \\
\hline 66 & RM 24542 & 9 & ATCCACAAGAGCACCGATGAGG & TGACCTGGTAGTGGTGAGTGTGC \\
\hline 67 & RM 24038 & 9 & GTCCGGGCAACAATAACAAAGC & CACCAACCGGGACTAAAGATGG \\
\hline 68 & RM 24780 & 9 & GACTAGCCAGCCAAGGTTTGAGC & TGCTGCATGTGTGTATGTGACTACG \\
\hline 69 & RM 25368 & 10 & TATAGTTAAGGGAGCCACGCAAGC & CCACCTCGTAAGAACATGGAGAACC \\
\hline 70 & RM25328 & 10 & CATTTGATCTGTCCGGCTCTAGG & ATTGCTAGTGGGTGATGATGTGG \\
\hline 71 & RM 6745 & 10 & GAAGCCTCCTTGATGTTGACTGG & CAACGATTATGCGTCCTTAGATGC \\
\hline 72 & RM 26329 & 11 & TAACCGGGACTAAAGATAGAGC & CTACGTCGAAATCGTAACTAGC \\
\hline 73 & RM 26086 & 11 & CAGCGCTTTGTACATCTCACTCC & GCATCCTCTGCACCTATTCTTGG \\
\hline 74 & RM 25970 & 11 & TGTGAATCTGACTTGCCAATCG & AAGGCTGTTATGTGTCTTGCAACC \\
\hline 75 & RM 27311 & 11 & TTACCAACCGGGACTAAAGATCG & CAATTCATAACGTCGGTCCTTCC \\
\hline 76 & RM 27034 & 11 & AGGCCCTCGCGTGTACATACC & ATCCGACCCACGGTAATCTGAGG \\
\hline 77 & RM 27101 & 11 & CGGGCACCATGGTATCTAATGC & ATGCTCGGTCCACAGAGAATAGG \\
\hline 78 & RM 28157 & 12 & GCTTAATTTCTGACAGACCAGTGC & GATCTAAACACAGCCTTCCTTGG \\
\hline 79 & RM 27840 & 12 & TTTGCGTGCTAGGGAGATTAGC & CATTATGTACTTACTCCСТCССТTCC \\
\hline 80 & RM 27406 & 12 & TGGTAGGTGTGCAATAGAAGTAGG & AATGCATGCAAACACAGTGG \\
\hline 81 & RM 3103 & 12 & CTGGAGTGGAGAAGAGAGAACAGG & TCTCCGCTCGGTTTCATCTAGG \\
\hline 82 & RM 28024 & 12 & CCGGGTGTTTGACCTAGTTTCC & CACGATGGTCGTCTTGTTACTGC \\
\hline 83 & RM 27864 & 12 & NA & NA \\
\hline 84 & RM 28464 & 12 & CTATATCCGCACCAATGGAAACC & TAGGAATTGCGACGAGTTTGTGG \\
\hline 85 & RM 27861 & 12 & TGCTAGCATGCTCATCCACTTGC & AATTGCCTCTGTAATACCGCCCTTCC \\
\hline 86 & RM 27905 & 12 & AACACAATTCACCGGTCCTTAGC & ATCGATCTTTAGTCCCGGATTGG \\
\hline 87 & RM 27882 & 12 & NA & NA \\
\hline 88 & JGT 01-16.2 & 1 & TGTTGGCTGAATGATTGATGGATAGA & GGACCTATGTGGCACGAATTTGAA \\
\hline 89 & JGT 01-23.1 & 1 & CGGCGTAGGAGTATTTGTAGGAAGTT & GCGGCAATGATGATGGTGGA \\
\hline 90 & JGT 01-29.9 & 1 & TCGATACTTTCGGAAATGGGAAGG & GCTAGCTCTAACATCCAAGCTTCC \\
\hline 91 & JGT $02-35.2$ & 2 & CGGGGCGAATCGAGGTCAG & TCGCGGTGGATTAGATGCCTC \\
\hline 92 & JGT 02-9.8 & 2 & GAGCGTTTTAAGGAAGCCACATG & CACCGAGTCCCAAGCTGATGA \\
\hline
\end{tabular}




\begin{tabular}{|c|c|c|c|c|}
93 & JGT $03-0.01$ & 3 & CCGATGCACCAACACCCTCAC & GACGCATGGTGAGTTTGGAGTGAT \\
\hline 94 & JGT $03-36.1$ & 3 & GCGGCAACACGACCAGCTT & CATCCTGAGTTTTGAGAAGCACCATA \\
\hline 95 & JGT $03-29.2$ & 3 & CCTGTAACCTGAACGGAGAGAGTA & TGAAAAAAAAAAGTCTCCCAATGTC \\
\hline 96 & JGT $04-11.5$ & 4 & AGGGCTCGTAGAAAGTGTCCAATTAG & CCGCTGGGTATTGCCTAACTG \\
\hline 97 & JGT $04-28.5$ & 4 & TTGAATAGATCGCACCGTTGAC & AAAAGGGCAGCTACGTCTGAGC \\
\hline 98 & JGT $04-34.9$ & 4 & ACGAACTCTAGTCCCTCCGCTC & ACGACATTATCCTAACACCACG \\
\hline 99 & JGT $05-05$ & 5 & TGTAGGAAGACATGTGGCAGCT & TACAACTAGATGCAATGGCACC \\
\hline 100 & JGT $05-7.8$ & 5 & ACGGTGAGATTAGGTATTGCCA & AGTTCGGACAAGGGGGAGTACT \\
\hline 101 & JGT $06-18.1$ & 6 & GAAGAACGTGGTTTGCATCGT & TGTACCGTCGGCGAAGAACGCACC \\
\hline 102 & JGT 0722.7 & 7 & AAGGGGTTATTGATTTAGTCCATTT & AGGGGTTGAACTTTGATGCTAATATG \\
\hline 103 & JGT $07-22.8$ & 7 & TGGCGATCTAGGAGCGTCTGT & TGTAAACATTTCAAAAGGGCACTAA \\
\hline 104 & JGT $08-19.5$ & 8 & TTCTGAAAAAGCTCTGACCAAGC & ACTAGCTACATGCTGCAGTGCAT \\
\hline 105 & JGT $08-10.2$ & 8 & GTACTAAACAGATGGAGCAGTACG & GTGGTACTGCTAATTAGGGGGTA \\
\hline 106 & JGT $08-16.7$ & 8 & GAAAGAATTTGTTCCCACGACCTC & GCTACAATTGCATGCTGGATGTCG \\
\hline 107 & JGT $09-4.8$ & 9 & CGGGATACTAACAGCAAGCAAAT & TCTCGCACCACTAACAGGGAACA \\
\hline 108 & JGT $10-0.3$ & 10 & TGGCGATTTAGGAGCATTTGTAGAA & GCCAACGACCACTCTCACCCTAC \\
\hline 109 & JGT $10-4.3$ & 10 & TGGCGACTTAGGAGCGTTTGTAG & GCGGCACTTCCCCAAACAA \\
\hline 110 & JGT $11-16.3$ & 11 & GGCGGCGTATTAGCGTTGTA & AGGTTCTAGCCCATGTTAAATCTTCT \\
\hline 111 & JGT $12-20.2$ & 12 & CTTACATGGATATGCACATCGAGC & CAAGGGAGCACACGAACAACAG \\
\hline 112 & JGT $12-7.7$ & 12 & GAGCGTTTTGAGGAAGTTTAATGGAC & GGCACCGTTTGATTTAGATTATTACC \\
\hline 113 & JGT $12-25.9$ & 12 & GGCGGCATAGGAGTGTTTGTAG & CGCGGTGGTCGGGATGAG \\
\hline
\end{tabular}

In the present study, DNA from 27 rice genotypes including Samba Mahsuri and other popular fine-grain type rice varieties developed by ANGRAU, Hyderabad, India were subjected to SSR marker analysis for identification of genotype specific markers/marker combinations for Samba Mahsuri. We have identified genotype specific SSR markers for Samba Mahsuri which can distinguish this variety from other medium-grain type slender grain types. The results obtained in the study are presented and discussed below.

Of the 113 SSR markers utilized in the present study, 26 displayed polymorphism among the 27 rice genotypes analyzed and amplified a total of 74 alleles with an average of 2.84 alleles per marker. The number of alleles detected by the polymorphic SSR markers varied from 2 to 5 . These include five alleles with one marker (RM16649), four alleles each with 2 markers (RM12469 and JGT07-22.8), three alleles each with 15 markers (RM14250, RM26329, RM15630,
RM6745, RM400, RM 10936, RM16153, RM16592, RM23237, RM14140, JGT0116.2, JGT03-29.2, JGT 12-20.2, JGT11-16.3, JGT04-11.5) and two alleles each with 8 markers (RM24542, RM27840, RM19426, RM11229, JGT03-36.1, JGT02-9.8, JGT030.01 and JGT10-4.3).

The SSR marker JGT11-16.3, targeting a $(\text { GATA })_{n}$ motif, clearly distinguished Samba Mahsuri from other medium slender varieties except MTU 2077, MTU 2067, Early samba, and WGL 14 by amplifying a 139 bp fragment, whereas another SSR marker RM19426 clearly distinguished Samba Mahsuri from the above mentioned four medium slender rice varieties by amplifying a $272 \mathrm{bp}$ fragment. When these two markers were pre-PCR multiplexed, they could clearly distinguish Samba Mahsuri from all the other rice varieties and hence the fragments amplified by both these markers (i.e. in combination) could be considered as a molecular ID for Samba Mahsuri (Figure 1). This can be considered the first step towards 
DNA fingerprinting of elite, quality rice varieties like Samba Mahsuri and thus protect the IPR of the institutions developing such unique and well accepted varieties. Followed (Sundaram et al., 2008) a similar multiplexing approach for checking the purity of hybrid rice parental lines. Based on the results of the present study, it can be concluded that SSR markers can be efficiently used to generate locus specific allelic information which can serve as molecular IDs for elite rice varieties like Samba Mahsuri

\section{Acknowledgement}

Directorate of rice research, Acharya. N G Ranga Agricultural University

\section{References}

Nandakumar N, Singh AK, Sharma RK, Mohapatra T, Prabhu KV, Zaman FU (2004). Molecular Wngerprinting of hybrids and assessment of genetic purity of hybrid seeds in rice using microsatellite markers. Euphytica 136:257-264

Qian Q, Chen H, Sun Z X and Zhu L H (1996). The study on determining true and false hybrid rice II You 63 Using RAPD molecular markers. Chinese Journal of Rice Science, 10: 241-242.

Rajendrakumar P, Akshaya Kumar Biswal, Kannabiran Sakthivel, Maganti Sheshu Madhav, Chirravuri Neeraja Sena M. Balachandran, Kommoju Srinivasarao Podishetty Natarajkumar Yadla Hari Kalidindi Sujatha Raman M (2009). Targeting (GATA)n repeat motifs in rice. Euphytica. DOI 10.1007/s10681-009-9964-x.

Sundaram R M, Naveenkumar B, Biradar S K, Balachandran S M, Mishra B, Ilyas Ahmed M, Viraktamath B C, Ramesha M S and Sarma N P (2008). Identification of informative SSR markers capable of distinguishing hybrid rice parental lines and their utilization in seed purity assessment. Euphytica, 163: 215-224.

Wang G S, Csatigline S, Zhang J, Fu R, Ma J $\mathrm{S}$, Wenbin L, Youngrusuh and Sala F. (1994). Hybrid rice (Oryza sativa L.): Identification and parentage determination by RAPD fingerprinting. Plant Cell Reports, 14: 112-115.

\section{How to cite this article:}

Aruna Kumari, K., A. Prasad babu, Ch.V. Durga Rani and Sundaram, R.M. 2018. Identification of Genotype Specific Marker for Samba Mahsuri (BPT 5204) Variety. Int.J.Curr.Microbiol.App.Sci. 7(12): 2495-2501. doi: https://doi.org/10.20546/ijcmas.2018.712.284 